Fizika Nizkikh Temperatur, 2000, v. 26, Nos. 9/10, p. 918-922

\title{
Structure characteristics of methane-doped solid normal hydrogen
}

\author{
N. N. Galtsov, A. I. Prokhvatilov, and M. A. Strzhemechny \\ B. Verkin Institute for Low Temperature Physics and Engineering, National Academy of Sciences of Ukraine \\ 47, Lenin Ave., 61164 Kharkov, Ukraine \\ E-mail: strzhemechny@ilt.kharkov.ua \\ Received February 7, 2000
}

\begin{abstract}
Structure studies of the quantum crystal of solid normal hydrogen doped with methane and deuteromethane are carried out by powder x-ray diffraction within the temperature range 5 to $12 \mathrm{~K}$. The concentration of the dopants in the gas mixtures used for sample preparation by quench condensing is varied from $0.5 \%$ to $5 \%$. It is established that the equilibrium solubility of both methanes is substantially below the lower fraction indicated. The abnormally high apparent extra volumes per impurity may be ascribed to van der Waals complexes formed around isolated dopant particles.
\end{abstract}

PACS: $67.80 . \mathrm{Mg}, \mathbf{6 4 . 7 5 . + \mathrm { g }}$

\section{Introduction}

Recently, solid hydrogen with the lowest possible concentration of the orthomodification was suggested [1] as a promising material for matrix isolation. Optical spectra of molecules embedded in solid hydrogen allow investigation of rather intimate quantum-crystal effects in the dynamics of these matrix-isolated molecules. There is also another interesting aspect, namely, the possibility of looking into some properties of the hydrogen matrix itself, in particular, into the response of the nearestneighbor environment to the introduction of a single molecular impurity. Additional interest in these solid-state issues comes from the fact that solid hydrogen is a quantum crystal, and all the solidstate renormalizations should take account of this circumstance. The recent work of Momose and coworkers [2-4] on the infrared spectra of the methanes $\mathrm{CH}_{4}$ and $\mathrm{CD}_{4}$, matrix isolated in parahydrogen, provides a good basis for a detailed study of the above effects. In particular, they fitted their spectra within the rigid lattice approach and extracted the values of the crystal field parameters and the renormalized rotational constants. For complete understanding of the effects observed, a few issues still need theoretical consideration and comparison with the relevant experiment. First, quantum-crystal renormalization of the parameters (and, possibly, of the form) of the noncentral interaction potential of the methane molecule with its matrix environment. Second, calculation of the displacements of nearest neighbors within Nosanow's [5] or more advanced [6,7] approaches. These displacements are important for the evaluation of the crystal field parameters, also to be renormalized in a quasistatic approximation for a soft lattice, which constitutes the third issue. Such evaluations, known for classical systems [8], require reconsideration for the case of quantum crystals. Finally, one more problem is the evaluation of the dynamic renormalization of the rotational constant of a single matrixisolated methane molecule. For the classical case such a procedure is known [9]. The difficulty of this program is aggravated by the fact that good energy surfaces for the interaction between $\mathrm{CH}_{4}$ and $\mathrm{H}_{2}$ are at present unavailable.

It is obvious that, however precise, the optical data give information on the rotational spectrum of the matrix-isolated molecule but do not tell directly about the actual geometry of the relaxed lattice around the impurity, which can be inferred from the known or assumed potentials. In this respect, structure studies, in which some independent conclusions about the response of the lattice surrounding can be drawn directly, may be treated as complementary to optical measurements. This was our main motivation of the work reported here.

An additional motivation of this structure study was to see whether the methane molecule in solid 
hydrogen exhibits the effect of abnormal excess volume, which is well observable, for example, in the $\mathrm{Ne}-\mathrm{H}_{2}$ system $[10,11]$ and cannot be observed in some other dilute hydrogen-based solid mixtures [12]. The strong effect of even weak doping with neon was attributed to the formation of van der Waals complexes $\mathrm{Ne}\left(\mathrm{H}_{2}\right)_{n}$. From optical and x-ray studies at high pressures and room temperature [13] it follows that the $n-\mathrm{H}_{2}-\mathrm{CH}_{4}$ system exhibits numerous stoichiometric compositions in the solid. We thought that some related effects might be felt at low temperatures in molar volumes as a function of the impurity concentration.

\section{Experimental}

Experiments were carried out on a DRON-3M powder $\mathrm{x}$-ray diffractometer equipped with a liquid helium cryostat. Diffractometer control as well as data collection and processing were automated. The samples were prepared by quench condensing gas mixtures of known composition on a flat copper substrate at a temperature close to $5 \mathrm{~K}$. Normally, we obtained polycrystalline samples about $0.1 \mathrm{~mm}$ thick with grain sizes $10^{-4}-10^{-5} \mathrm{~mm}$. Condensation of every batch resulted in a heating of the substrate by 3 to $4 \mathrm{~K}$. The purity of the gases used for sample preparation were: normal hydrogen $99.999 \%$, methane and deuteromethane $99.99 \%$. The error in the impurity concentration measured by means of the $P-V-T$ method did not exceed $5 \%$ of the rated impurity fraction. The concentration ranges studied are $0.5 \%$ to $5 \%$ for $\mathrm{CH}_{4}$ and $1 \%$ to $5 \%$ for $\mathrm{CD}_{4}$. The temperature ranges are from $5 \mathrm{~K}$ to the melting points of the hydrogen matrices. Temperature stabilization and measurement were performed to within $\pm 0.05 \mathrm{~K}$. The lattice parameters were determined to within $\pm 0.02 \%$.

\section{Results and discussion}

Typical diffraction patterns for the region of smaller diffraction angles, where the most intense reflections from the solid mixtures $n-\mathrm{H}_{2}+0.5 \% \mathrm{CH}_{4}$ and $n-\mathrm{H}_{2}+1 \% \mathrm{CD}_{4}$ were observed, are reproduced in Figs. 1 and 2. Both plots include patterns from as-prepared samples at $5 \mathrm{~K}$ and from the same samples warmed up to and kept for 30 minutes at $12 \mathrm{~K}$. Figure 2 also shows a diffraction pattern taken after a cooldown to $5 \mathrm{~K}$ following the above annealing. The patterns from both systems exhibit a very strong (002) texture with the close packed hexagonal layers of $n-\mathrm{H}_{2}$ being predominantly parallel to the substrate plane. The presence of me-

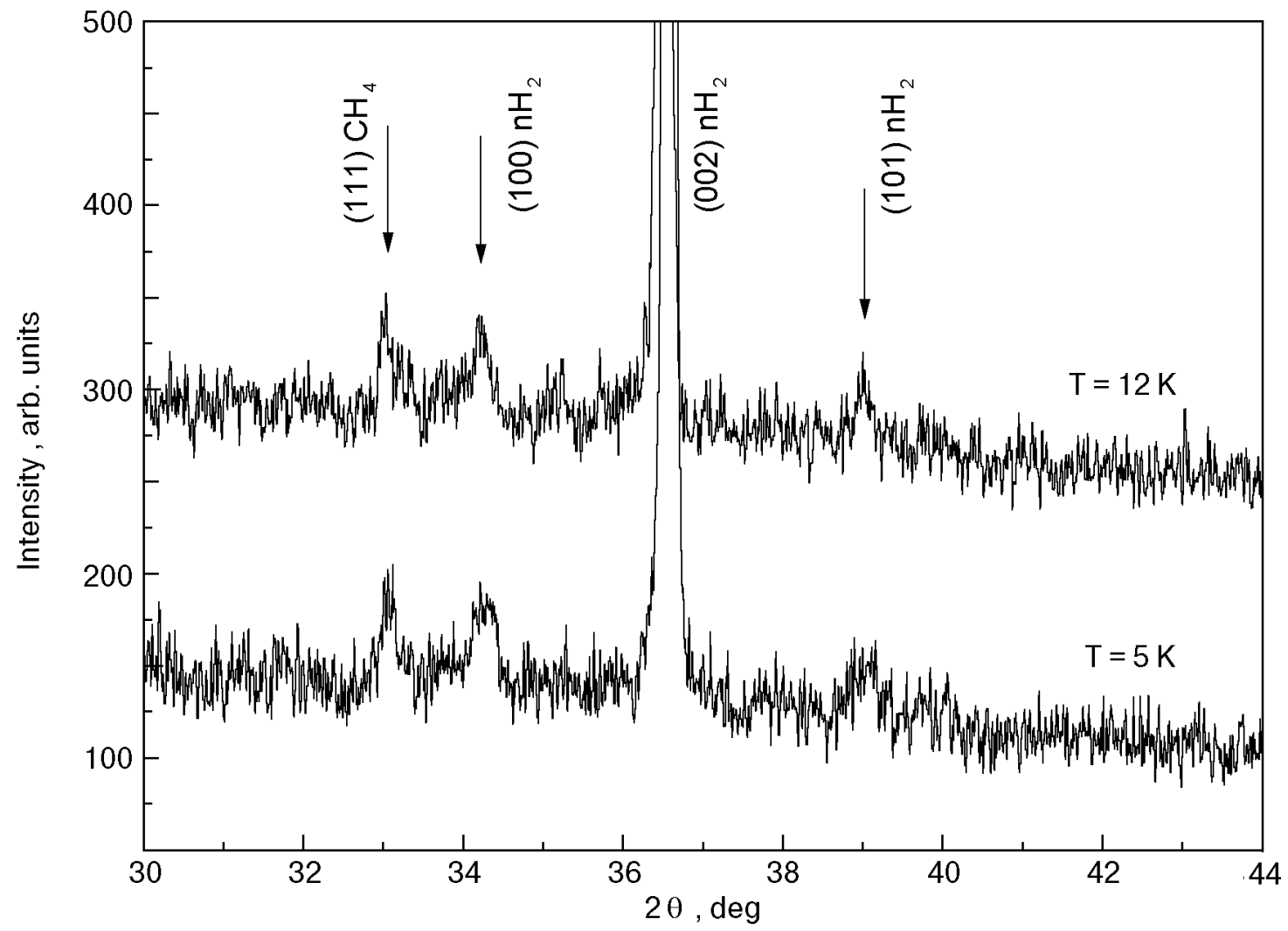

Fig. 1. X-ray powder diffraction patterns from $n-\mathrm{H}_{2}+0.5 \% \mathrm{CH}_{4}$ solid mixtures at the temperatures indicated. The most intense methane line (cubic 111) is well discernible. The arrows indicate Bragg reflections. Traces are shifted vertically to avoid overcrowding. The intensity counts are in arbitrary units but which are the same for all traces. 
thane impurities serves as a factor that stabilizes the texture obtained during quenching. The texture did not undergo noticeable changes when the crystals were warmed up to premelting temperatures and subsequently cooled down (see Fig. 2). Similar textures were observed previously when pure $n-\mathrm{H}_{2}$ or $p-\mathrm{H}_{2}$ samples were also quench condensed on cold substrates at liquid helium temperature [14,15]. But unlike doped crystals, warming up polycrystalline pure hydrogen samples to a few tenths of a Kelvin below $T_{m}$ usually resulted in an enhanced texture, viz., presumably due to recrystallization, the crystals exhibited single-crystal features: the reflection (002) grew in intensity by orders of magnitude, and the other lines disappeared. Subsequent cooldown did not change the patterns obtained at higher temperatures.

Even for methane and deuteromethane fractions about $1 \%$ and lower the diffraction patterns display the brightest (111) line of the respective fcc lattices of both $\mathrm{CH}_{4}$ and $\mathrm{CD}_{4}$ (see Figs. 1 and 2); this line grew in intensity as the dopant fraction was increased.

Heating of the samples entailed a noticeable shift of all visible reflections to smaller diffraction angles, which is caused by thermal expansion. When subsequently cooled down back to $5 \mathrm{~K}$ the positions of the lines were restored (see Fig. 2) completely, as inferred from calculated lattice parameters before and after warmup. It should be also added that the linewidths did not change after thermocycling. Such a behavior implies that the methane molecules have not been redistributed during the procedure.

Owing to the considerable molar volume difference between the $n$ - $\mathrm{H}_{2}$ matrix $\left(22.83 \mathrm{~cm}^{3} / \mathrm{mol}\right.$ [14]) and $\mathrm{CH}_{4}\left(30.27 \mathrm{~cm}^{3} / \mathrm{mol}\right.$ [16] $)$ or $\mathrm{CD}_{4}$ $\left(29.37 \mathrm{~cm}^{3} / \mathrm{mol}\right.$ [17]), formation of the solid mixtures should be accompanied by substantial lattice distortions around the impurities, which in turn must entail broader and weaker diffraction lines. In our experiments the reflections belonging to the matrix are narrow $\left(0.2^{\circ}\right.$ to $\left.0.4^{\circ}\right)$, comparable with those in pure normal hydrogen. This finding, together with the fact that we see reflections from a methane-rich phase, gives us grounds to conclude that the equilibrium solubility of the methanes in solid hydrogen is extremely low.

The molar volumes of the normal hydrogen matrix doped with the light and heavy methane isotopes are plotted in Figs. 3, $a$ and 3,b. One can see that within the entire concentration range studied, starting with fractions as low as $0.5 \%$ of $\mathrm{CH}_{4}$ and $1 \%$ of $\mathrm{CD}_{4}$, the molar volume of the matrix is virtually concentration independent. A similar independence holds for higher temperatures, 1 or $2 \mathrm{~K}$ below the melting point of $n-\mathrm{H}_{2}$. This result is in

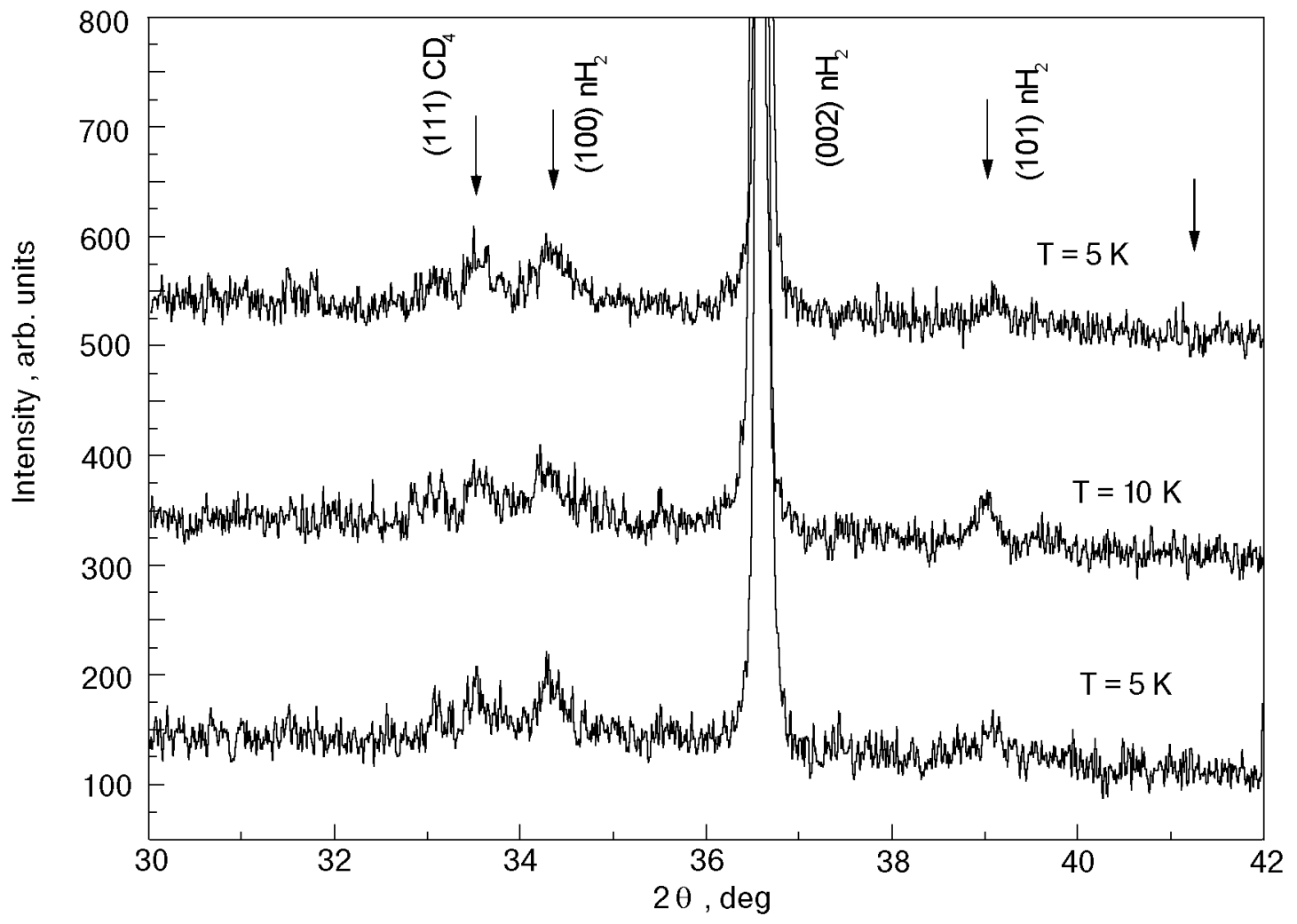

Fig. 2. X-ray powder diffraction patterns from $n-\mathrm{H}_{2}+1 \% \mathrm{CD}_{4}$ solid mixtures at the temperatures indicated. The pattern with the thick downward arrow is for the same sample cooled down to $5 \mathrm{~K}$ after a warmup to $12 \mathrm{~K}$. Other notations are as in Fig. 1. 

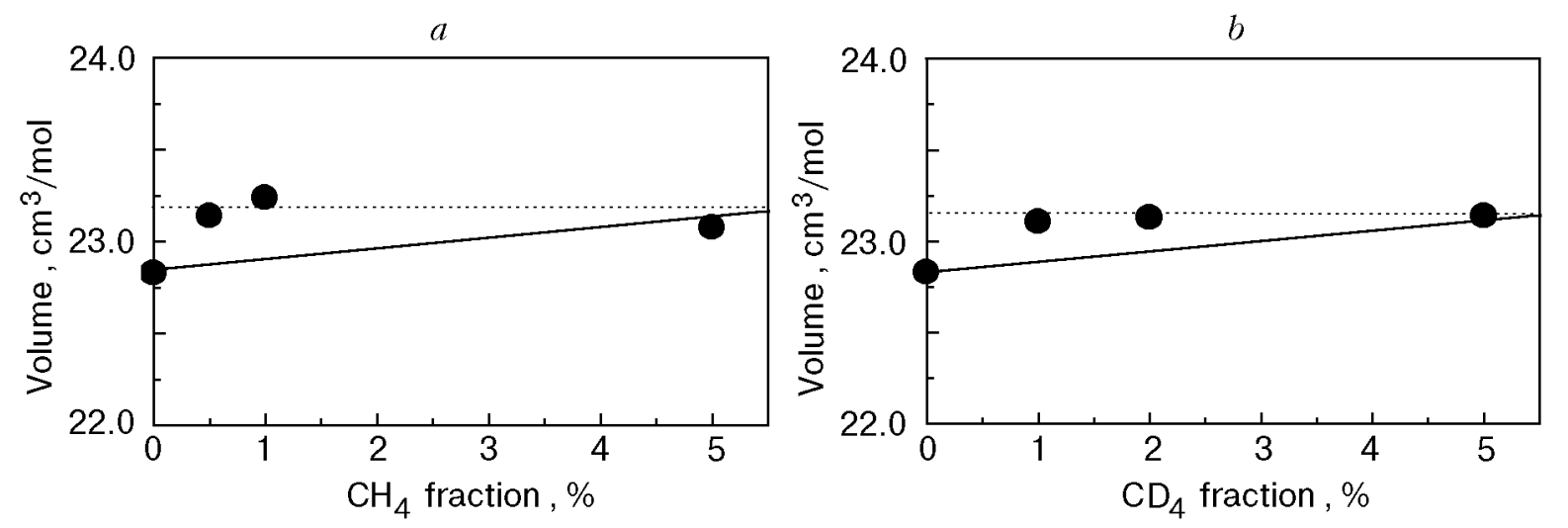

Fig. 3. The molar volumes of $n-\mathrm{H}_{2}-\mathrm{CH}_{4}(a)$ and $n-\mathrm{H}_{2}-\mathrm{CD}_{4}($ b $)$ alloys versus the methane concentration as calculated from the patterns at $5 \mathrm{~K}$. The impurity concentrations correspond to their content in the gas mixtures from which the polycrystal samples were prepared.

line with the conclusion formulated above that the equilibrium solubility of methane molecules in hydrogen is very low, at a level of few tens of ppm.

Notwithstanding the low solubility, the effect of this molecular impurity on the lattice parameters of the matrix is abnormally strong, just like in the case of neon. Introduction of a small amount of methane results in an increase of the molar volume of the matrix by $1.3 \%$. If these solid solutions were ideal, the dissolution of $1 \%$ of methane would have increased the volume of the matrix by $0.3 \%$ (for deuteromethane the figure is $0.28 \%$ ). If we assume that half of the dopant is in dispersed form, we would obtain for the reduced extra volume per impurity, $\left[V(x)-V_{0}\right] /\left(x V_{0}\right)$, a very large value of around $5.2 \pm 1.8$. We think that the effect observed in the normal hydrogen matrix might be, at least in part, due to van der Waals complexes formed around the methane impurities, as has been assumed for the presumably similar effect in neon-doped normal hydrogen [8].

We note that the introduction of methane molecules into the hydrogen lattice entails an effect on the measured molar volume of the matrix, which is comparable to that due to thermal expansion over the entire range of existence of the solid phase $(\Delta V / V=1.4 \%$ [16]) or due to conversion ( $\Delta V / V=1.04 \%$ [17] $)$. This must lead to noticeable changes in the thermodynamic and structural characteristics of hydrogen-methane alloys. As to the latter, we found that admixture of methane leads not only to larger molar volumes but tends to change the $c / a$ ratio closer to its ideal value of 1.633. At $5 \mathrm{~K}$ the lattice parameters of the $1 \%$ $\mathrm{CH}_{4}$ in $\mathrm{H}_{2}$ mixture are: $a=3.791 \AA$; $c=6.176 \AA$; $c / a=1.630 ; V=23.12 \mathrm{~cm}^{3} / \mathrm{mol}$. These values are close to those found for parahydrogen [15]. Deuteromethane produces a similar effect.

In conclusion, powder $\mathrm{x}$-ray studies of the lattice parameters of solid normal hydrogen doped with $\mathrm{CH}_{4}$ and $\mathrm{CD}_{4}$ at fractions down to $0.5 \%$ have shown that the equilibrium solubility of both methanes is very low. An abnormally strong effect of methane impurities on the molar volume of the normal hydrogen matrix has been documented. The effect is tentatively ascribed to a specific quantumcrystal response of the lattice to the formation of van der Waals complexes around the methane impurities.

1. T. Momose and T. Shida, Bull. Chem. Soc. Jpn. 71, 1 (1998).

2. T. Momose, J. Chem. Phys. 107, 7695 (1997).

3. T. Momose, M. Miki, T. Wakabayashi, and T. Shida, $J$. Chem. Phys. 107, 7707 (1997).

4. H. Hoshina, T. Wakabayashi, T. Momose, and T. Shida, J. Chem. Phys. 110, 5728 (1997).

5. L. H. Nosanow, Phys. Rev. 146, 120 (1966).

6. H. Horner, Z. Phys. 242, 432 (1971).

7. T. R. Koehler, Phys. Rev. 165, 942 (1968).

8. S. E. Kalnoi, M. A. Strzhemechny, V. V. Sumarokov, and Yu. A. Freiman, Fiz. Nizk. Temp. 13, 809 (1987) [Sov. J. Low Temp. Phys. 13, 462 (1987)].

9. T. N. Antsygina and V. A. Slusarev, Teor. Mat. Fiz. 77, 234 ( 1980) [in Russian].

10. A. S. Barylnik, A. I. Prokhvatilov, M. A. Strzhemechny, and G. N. Shcherbakov, Fiz. Nizk. Temp. 19, 625 (1993) [Low Temp. Phys. 19, 447 (1993)].

11. A. S. Barylnik, A. I. Prokhvatilov, and G. N. Shcherbakov, Fiz. Nizk. Temp. 21, 787 (1995) [Low Temp. Phys. 21, 607 (1995)].

12. G. N. Shcherbakov, A. I. Prokhvatilov, M. A. Strzhemechny, and A. S. Barylnik, Abstracts, Cryocrystals-2 Intern. Conf., Sept. 1997, Vroclaw (1997).

13. M. S. Somayazulu, L. W. Finger, R. J. Hemley, and H. K. Mao, Science 271, 1400 (1996). 
14. I. N. Krupskii, A. I. Prokhvatilov, and G. N. Shcherbakov, Fiz. Nizk. Temp. 9, 858 (1983) [Sov. J. Low. Temp. Phys. 9, 446 (1983)].

15. I. N. Krupskii, A. I. Prokhvatilov, and G. N. Shcherbakov, Fiz. Nizk. Temp. 9, 83 (1983) [Sov. J. Low. Temp. Phys. 9, 42 (1983)].
16. A. I. Prokhvatilov and A. P. Isakina, Fiz. Nizk. Temp. 9 419 (1983) [Sov. J. Low. Temp. Phys. 9, 213 (1983)].

17. A. I. Prokhvatilov and A. P. Isakina, Phys. Status Solidi A78, 147 (1983). 